\title{
Clinical Translation of an Albumin-Binding PET Radiotracer ${ }^{68}$ Ga-NEB
}

\author{
Jingjing Zhang ${ }^{1,2}$, Lixin Lang ${ }^{2}$, Zhaohui Zhu ${ }^{1}$, Fang $\mathrm{Li}^{1}$, Gang Niu ${ }^{2}$, and Xiaoyuan Chen ${ }^{2}$ \\ ${ }^{I}$ Department of Nuclear Medicine, Peking Union Medical College Hospital (PUMCH), Chinese Academy of Medical Sciences and \\ Peking Union Medical College (CAMS and PUMC), Beijing, China; and ${ }^{2}$ Laboratory of Molecular Imaging and Nanomedicine, \\ National Institute of Biomedical Imaging and Bioengineering, National Institutes of Health, Bethesda, Maryland
}

Suitably labeled Evans blue dye has been successfully applied to evaluate cardiac function, vascular permeability, and lymphatic imaging in preclinical settings. This study documented the first-inhuman application of ${ }^{68} \mathrm{Ga}-1,4,7$-triazacyclononane- $N, N^{\prime}, N^{\prime \prime}$-triacetic acid (NOTA)-NEB. Methods: The NOTA-conjugated truncated form of Evans blue, NEB, was labeled with ${ }^{68} \mathrm{Ga}$ and tested in BALB/C mice for dynamic PET and ex vivo biodistribution studies. Three healthy volunteers (2 men and 1 woman) underwent 90-min wholebody dynamic PET. The absorbed doses for major organs and whole body were calculated using OLINDA/EXM software. Eleven patients with focal hepatic lesions diagnosed by enhanced CT or MR imaging were subjected to whole-body PET/CT acquisitions at $30 \mathrm{~min}$ after intravenous injection of $111-148 \mathrm{MBq}(3-4 \mathrm{mCi})$ of ${ }^{68} \mathrm{Ga}-\mathrm{NEB}$. Results: NEB dye was labeled with ${ }^{68} \mathrm{Ga}$ (half-time, $68 \mathrm{~min}$ ) with high yield and purity. After intravenous injection, ${ }^{68} \mathrm{Ga}-\mathrm{NEB}$ formed a complex with serum albumin, thus most of the radioactivity was retained in blood circulation. The tracer was demonstrated to be safe in both healthy volunteers and recruited patients without side effects or allergies. Among the 11 patients, hemangiomas showed much higher ${ }^{68} \mathrm{Ga}-\mathrm{NEB}$ signal intensity than the surrounding normal hepatic tissues, whereas no apparent difference between lesions and hepatic tissues was identified on ${ }^{18} \mathrm{~F}$-FDG PET. All other focal hepatic lesions including hepatocellular carcinoma, hepatic cysts, and neuroendocrine tumor liver metastases showed negative ${ }^{68} \mathrm{Ga}-\mathrm{NEB}$ contrast to hepatic tissues. Conclusion: As a blood-pool imaging agent, ${ }^{8} \mathrm{Ga}-$ NEB is safe to use in the clinic, and our preliminary studies demonstrate the value of differentiating hepatic hemangioma from other benign or malignant focal hepatic lesions. Easy labeling with different positron emitters of various half-lives, excellent pharmacokinetics, and imaging quality warrant further clinical applications of NEBbased PET tracers.

Key Words: truncated Evans blue; albumin; blood pool; first-inhuman; hemangioma

J Nucl Med 2015; 56:1609-1614

DOI: 10.2967/jnumed.115.159640

Received Apr. 20, 2015; revision accepted Jul. 17, 2015.

For correspondence or reprints contact either of the following:

Fang Li, Peking Union Medical College, 9 Dongdan 3rd Alley, Dongcheng,

Beijing, China.

E-mail: lifang@pumch.cn

Xiaoyuan Chen, National Institutes of Health (NIH), 35A Convent Dr.,

GD937, Bethesda, MD 20892-3759.

E-mail: shawn.chen@nih.gov

Published online Aug. 6, 2015.

COPYRIGHT (c) 2015 by the Society of Nuclear Medicine and Molecular Imaging, Inc.
A ngiography plays a critical role in screening, diagnosis, treatment planning, and prognosis in vascular diseases. With blood-pool contrast agents, CT and MR imaging angiography provide excellent spatial and temporal resolution of arteries and veins, thus replacing the invasive conventional angiography (1). However, the relatively low sensitivity of CT and MR imaging requires administration of large quantities of contrast agents, which increases the risks of allergic reaction or nephrotoxicity (2).

Radionuclide angiography, with ${ }^{99 \mathrm{~m}} \mathrm{Tc}$-labeled red blood cells (RBCs) as the dominant imaging agent, has been used clinically as early as 1958 for determining total blood and plasma volumes, cardiac output, protein turnover studies, heart and great vessel delineation, and localization of cerebral neoplasms (3). Compared with SPECT, PET is more sensitive and has higher spatial resolution with clinical scanners.

A few PET blood-pool imaging tracers have been introduced for labeling RBCs. For example, carbon monoxide containing either ${ }^{11} \mathrm{C}$ or ${ }^{15} \mathrm{O}$ has been used to label RBCs for PET (4). However, because of their short half-lives $\left(20.4 \mathrm{~min}\right.$ for ${ }^{11} \mathrm{C}$ and $2.05 \mathrm{~min}$ for ${ }^{15} \mathrm{O}$ ), these tracers can be used only in centers with an in-house cyclotron. Furthermore, the need for administration of the gaseous form of carbon monoxide by inhalation necessitates sophisticated equipment for human as well as animal studies (5). The use of RBCs for blood-pool imaging also presents significant risks to both the patient receiving the product and the operator handling radiolabeled RBCs. Thus, there is a critical need for a clinically practicable PET blood-pool imaging agent for angiography.

Instead of RBCs, serum albumin has been labeled with various positron-emitting radionuclides for PET (6-10). The general strategy was to conjugate albumin with radioisotopes in vitro and then inject the labeled albumin intravenously for imaging. However, the unfavorable pharmacokinetics of these albumin-based tracers, such as high liver accumulation, suggests that the albumin structure may have been partially disrupted during the bioconjugation and labeling process. Moreover, in vitro protein labeling is time consuming and requires multiple steps of purification and quality control.

Alternatively, we have developed a method for in vivo labeling of the endogenous albumin by conjugating a cyclic chelator, 1,4,7-triazacyclononane- $N, N^{\prime}, N^{\prime \prime}$-triacetic acid (NOTA), on the truncated form of Evans blue (EB), denoted as NEB (11). EB dye has high affinity for serum albumin and has been used clinically to determine blood volume $(12,13)$. Although recently discontinued in clinical practice, EB is still used as a sensitive marker of protein leakage from the vascular lumen in a variety of tissues during inflammation and traumatic injury $(14,15)$. The radiolabeling 
of ${ }^{18} \mathrm{~F}$ was achieved by the formation of ${ }^{18} \mathrm{~F}$-aluminum fluoride complex (16-18). The entire labeling process takes about 20-30 min without the need of high-performance liquid chromatography purification (11). After intravenous injection, ${ }^{18} \mathrm{~F}-\mathrm{AlF}-\mathrm{NEB}$ complexed with serum albumin quickly, and thus most of the radioactivity remained in the blood circulation. ${ }^{18} \mathrm{~F}-\mathrm{AlF}-\mathrm{NEB}$ has been successfully applied to evaluate cardiac function in a myocardial infarction model and vascular permeability in inflammatory and tumor models (11). In a more recent study, we applied ${ }^{18} \mathrm{~F}-\mathrm{AlF}-\mathrm{NEB}$ along with the EB dye for lymphatic imaging after local injection (19). In view of the great success of NEB in preclinical investigations, we moved forward to clinical applications. Instead of ${ }^{18} \mathrm{~F}$, we used ${ }^{68} \mathrm{Ga}$ as the positron emitter, because with ${ }^{68} \mathrm{Ga}$ the cyclotron was nonessential and because of the feasibility of kit formulation (20).

\section{MATERIALS AND METHODS}

\section{Preparation of ${ }^{68} \mathrm{Ga}-\mathrm{NEB}$}

NEB was synthesized according to a method described in our previous publication (11). ${ }^{68} \mathrm{Ga}$ was eluted from a ${ }^{68} \mathrm{Ge} /{ }^{68} \mathrm{Ga}$ generator (ITG) using $0.05 \mathrm{M} \mathrm{HCl}$ and mixed with $1.25 \mathrm{M} \mathrm{NaOAc}$ buffer to adjust the $\mathrm{pH}$ value to 4.0. The mixture was then directly transferred to a $1-\mathrm{mL}$ plastic tube containing $30 \mu \mathrm{g}$ of NEB. After shaking, the mixture was incubated in a heating block at $100^{\circ} \mathrm{C}$ for $10 \mathrm{~min}$. Then the reaction mixture was cooled down, dissolved in sterile phosphatebuffered saline, and passed through a $0.22-\mu \mathrm{m}$ aseptic filtration membrane. The quality control was performed with analytic highperformance liquid chromatography and thin-layer chromatography (Bioscan, USA). $\mathrm{CH}_{3} \mathrm{OH}: \mathrm{NH}_{4} \mathrm{OAc}$ (v/v 1:1) was used as the developing solution for thin-layer chromatography. The radiochemical purity was greater than $95 \%$.

\section{Preclinical PET Imaging}

All animal studies were conducted in accordance with the principles and procedures outlined in the Guide for the Care and Use of Laboratory Animals (21) and were approved by the Institutional Animal Care and Use Committee of the Clinical Center, National Institutes of Health. For dynamic PET scans, 4 BALB/C mice were injected intravenously with $1.85 \mathrm{MBq}(50 \mu \mathrm{Ci})$ of ${ }^{68} \mathrm{Ga}-\mathrm{NEB}$ under isoflurane anesthesia. A 60-min list-mode acquisition was performed with an Inveon PET scanner (Siemens Preclinical Solutions). Images were reconstructed using the 2-dimensional ordered-subsets expectation maximum algorithm without attenuation or scatter correction. Regions of interest (ROIs) over major organs were drawn on decay-corrected whole-body coronal images for each PET scan, using vendor software (ASI Pro 5.2.4.0; Preclinical Solutions, Siemens). The radioactivity concentration (accumulation) within a tumor or an organ was obtained from mean pixel values within the multiple-ROI volume, after conversion of the values to $\mathrm{MBq} / \mathrm{mL} / \mathrm{min}$ using a conversion factor. The conversion to $\mathrm{MBq} / \mathrm{g} / \mathrm{min}$ assumed a tissue density of $1 \mathrm{~g} / \mathrm{mL}$. Imaging ROI-derived percentage injected dose per gram was calculated by dividing the ROIs by the administered activity (22).

\section{Volunteers and Patient Recruiting}

This first-in-human study was approved by the Institutional Review Board of Peking Union Medical College Hospital, Chinese Academy of Medical Sciences, and Peking Union Medical College. All subjects signed a written informed consent form and were informed of the benefits and possible risks of participating in the study. Three healthy volunteers ( 2 men and 1 woman) were enrolled to validate the safety of ${ }^{68} \mathrm{Ga}-\mathrm{NEB}$. No fasting, hydration, or other specific preparation was requested on the day of imaging. For all 3 healthy volunteers, the blood pressure, pulse, respiratory rate, and temperature were measured before and after injection of tracer ${ }^{68} \mathrm{Ga}-\mathrm{NEB}$. In addition, liver function, kidney function, routine blood, and urine tests were performed in 2 of the 3 volunteers before and $24 \mathrm{~h}$ after the scans. Any unusual or adverse clinical symptoms volunteers complained about or physical signs including the blood pressure, pulse, respiratory rate, temperature, liver function, kidney function, routine blood tests, and urine tests were included and recorded in the clinical evaluation. Any unusual or adverse clinical symptoms were recorded on the day of imaging and during the 2-wk follow-up period.

Eleven patients with hepatic space-occupying lesions were recruited. The inclusion criteria included the following: being identified with hepatic lesions by MR imaging or CT (the lesions were suspected as hemangioma, or hepatic malignancy, and needed differentiation from other benign diseases with surgery or biopsy); at least $18 \mathrm{y}$ old; and able to provide basic information and sign the written informed consent form. The final diagnosis was based on pathologic results of surgical removal or biopsy. Among the recruited patients, 4 patients were diagnosed with hemangioma, 5 with primary hepatic carcinoma, 1 with neuroendocrine tumor liver metastases, and 1 with hepatic cyst. All the recruited patients underwent surgery after the PET scans, as recommended by their clinicians.

\section{PET Imaging Procedures}

All 3 healthy volunteers underwent whole-body PET acquisitions (Biograph mCT PET/CT system; Siemens) at multiple time points after tracer injection. After the whole-body low-dose CT scan $(140 \mathrm{kV}$; $35 \mathrm{~mA}$; pitch, 1:1; layer, $5 \mathrm{~mm}$; layer spacing, $3 \mathrm{~mm}$; matrix, $512 \times 512$; field of view, $70 \mathrm{~cm}$ ), $111-148 \mathrm{MBq}(3-4 \mathrm{mCi})$ of ${ }^{68} \mathrm{Ga}-\mathrm{NEB}$ were injected intravenously. The whole body (from the top of the skull to the middle of the femur) of each volunteer was covered by 7 bed positions. The acquisition duration was $40 \mathrm{~s} / \mathrm{bed}$ position for the 5-, 10-, and 15min time points and $2 \mathrm{~min} /$ bed position for the 30-, 45-, 60-, 75-, and 90-min time points.

All patients with hepatic lesions underwent whole-body PET/CT acquisitions at $30 \mathrm{~min}$ after intravenous injection of 111-148 MBq (3$4 \mathrm{mCi}$ ) of ${ }^{68} \mathrm{Ga}-\mathrm{NEB}$, with each bed position lasting for $2 \mathrm{~min}$. The acquisition field covered from the top of the skull to the middle of the femur with 6 or 7 bed positions, depending on the height of the patient. An ${ }^{18} \mathrm{~F}-\mathrm{FDG}$ PET/CT image was acquired with the same patients within $1 \mathrm{wk}$.

\section{Image and Data Analysis}

A Siemens MMWP workstation was used for postprocessing. Visual analysis was used to determine the general biodistribution and the temporal and intersubject stability. The volume of interest of normal organs/tissues and concerned lesions was drawn on the serial images. The radioactivity concentration and standardized uptake value (SUV) in the volumes of interest were obtained through the software. All quantitative data were expressed as mean \pm SD.

The dosimetry calculation was performed according to the European Association of Nuclear Medicine dosimetry guidance (23). The decay-uncorrected time-activity curve was generated on the basis of the SUV of each organ. The SUVs were converted to MBq/MBq based on organ weight from the adult male phantom $(73.7 \mathrm{~kg}$ of body weight) and the adult female phantom (56.9 kg of body weight) provided by OLINDA/EXM (version 1.1; Vanderbilt University) $(24,25)$. The time-integrated activity coefficient (residence time) of each organ was determined by fitting the data using a biphase exponential model provided by the software. The urine bladder volume was determined by reconstructed PET/CT images, and the residence time was calculated by the trapezoidal method using GraphPad Prism (version 4.0; GraphPad Software, Inc.). The void time was set as $60 \mathrm{~min}$. The remainder of body was calculated for each time point as the decayed value of the original injected activity minus activity present in the identified source organs. Then the absorbed doses were calculated by entering the 


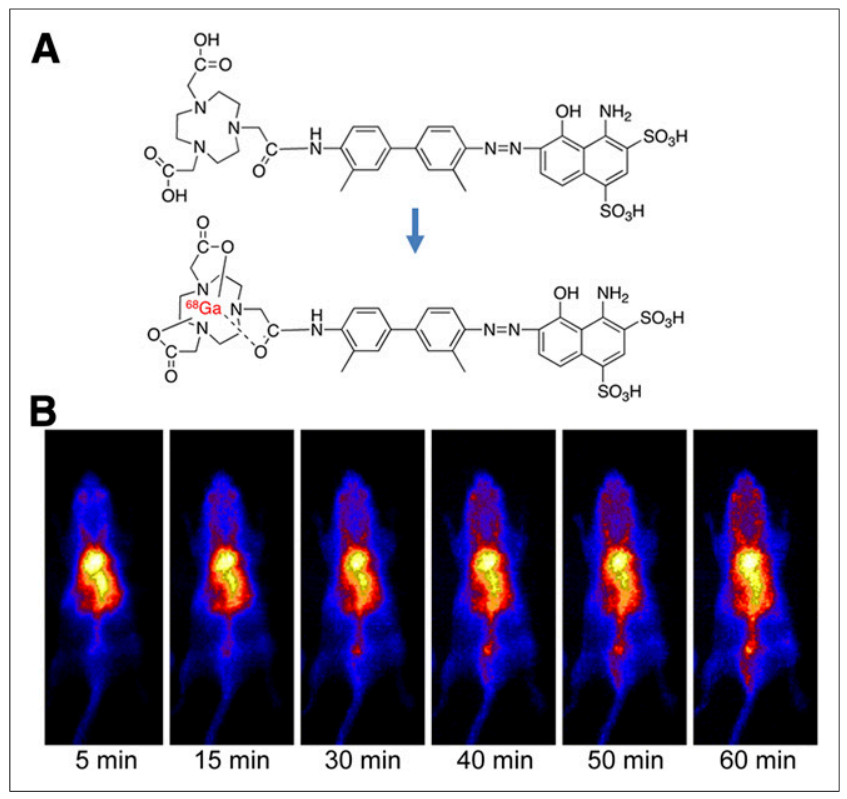

FIGURE 1. (A) Chemical structure of NEB and radiolabeling of NEB with ${ }^{68} \mathrm{Ga}$. (B) Representative coronal maximum-intensity-projection images of $60-$ min dynamic ${ }^{68} \mathrm{Ga}-\mathrm{NEB}$ PET of normal BALB/C mouse.

time-integrated activity coefficient for all source organs into OLINDA/ EXM for either a $73.7-\mathrm{kg}$ adult male or $56.9-\mathrm{kg}$ adult female.

\section{RESULTS}

\section{Chemistry and Radiochemistry}

The structure and radiolabeling of NEB with ${ }^{68} \mathrm{Ga}$ are presented in Figure 1A. The whole labeling process of ${ }^{68} \mathrm{Ga}-\mathrm{NEB}$ took about $30 \mathrm{~min}$, with a radiochemical purity of greater than $95 \%$ as determined by both analytic radio-high-performance liquid chromatography and radio-thin-layer chromatography. ${ }^{68} \mathrm{Ga}-\mathrm{NEB}$ showed good stability in mouse serum after 120 min of incubation (Supplemental Fig. 1; supplemental materials are available at http://jnm. snmjournals.org).

\section{In Vivo Dynamic PET Imaging and Biodistribution in Normal Mice}

To investigate the pharmacokinetics of ${ }^{68} \mathrm{Ga}-\mathrm{NEB}$, we performed 1-h dynamic PET in healthy BALB/C mice. After tail vein injection, most of the radioactivity from ${ }^{68} \mathrm{Ga}-\mathrm{NEB}$ was retained in the blood circulation, including the ventricles of the heart, major arteries, and blood-enriched organs, during the entire period of observation (Fig. 1B; Supplemental Fig. 2).

To estimate the safe dose for clinical use, we performed an ex vivo biodistribution study in normal BALB/C mice. The absorbed doses for major organs and whole body were then extrapolated to an adult human male of a body weight of $73.7 \mathrm{~kg}$ using OLINDA/EXM software. The mean dose range from 5 mice at each time point is listed in Supplemental Table 1. The kidneys received the highest absorbed doses (mean absorbed dose, 0.104-0.135 mSv/MBq), resulting from abundant blood supply and tracer excretion through the renal urinary tract. The mean effective dose of ${ }^{68} \mathrm{Ga}-\mathrm{NEB}$ was $0.0151-0.0159 \mathrm{mSv} / \mathrm{MBq}$. With an injected dose of $185 \mathrm{MBq}(5 \mathrm{mCi})$, the patient would be exposed to an effective radiation dose of $2.94 \mathrm{mSv}$, which is much lower than the dose limit of $20 \mathrm{mSv}$ for the second risk category

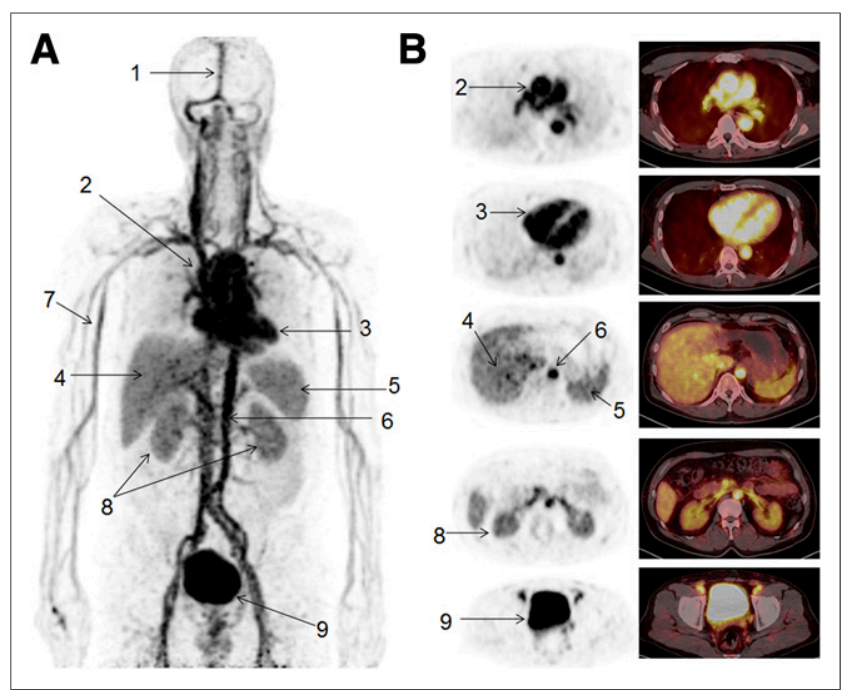

FIGURE 2. (A) Coronal maximum-intensity-projection image of male volunteer at $30 \mathrm{~min}$ after intravenous administration of $138.75 \mathrm{MBq}(3.75 \mathrm{mCi})$ of ${ }^{68} \mathrm{Ga}-\mathrm{NEB}$. Principal organs and regions of uptake are labeled: superior sagittal sinus (1), arch of aorta (2), cardiac ventricles (3), liver (4), spleen (5), abdominal aorta (6), limb vessels (7), kidneys (8), and bladder (9). (B) Corresponding axial PET and PET/CT fusion images at key levels to reflect arch of aorta, cardiac ventricles, liver and spleen, kidneys, and bladder.

defined by the 2007 International Commission on Radiological Protection (26).

\section{Healthy Volunteers and Dosimetry}

With a mean injected dose of $139.5 \pm 10.36 \mathrm{MBq}(3.77 \pm 0.28$ $\mathrm{mCi}$ ), no adverse symptoms were noticed or reported during the entire procedure and 2-wk follow-up, demonstrating the safety of the tracer. A representative 2-dimensional projection PET image acquired at $30 \mathrm{~min}$ after intravenous administration of ${ }^{68} \mathrm{Ga}-\mathrm{NEB}$ is presented in Figure 2. Cardiac ventricles, major arteries, and

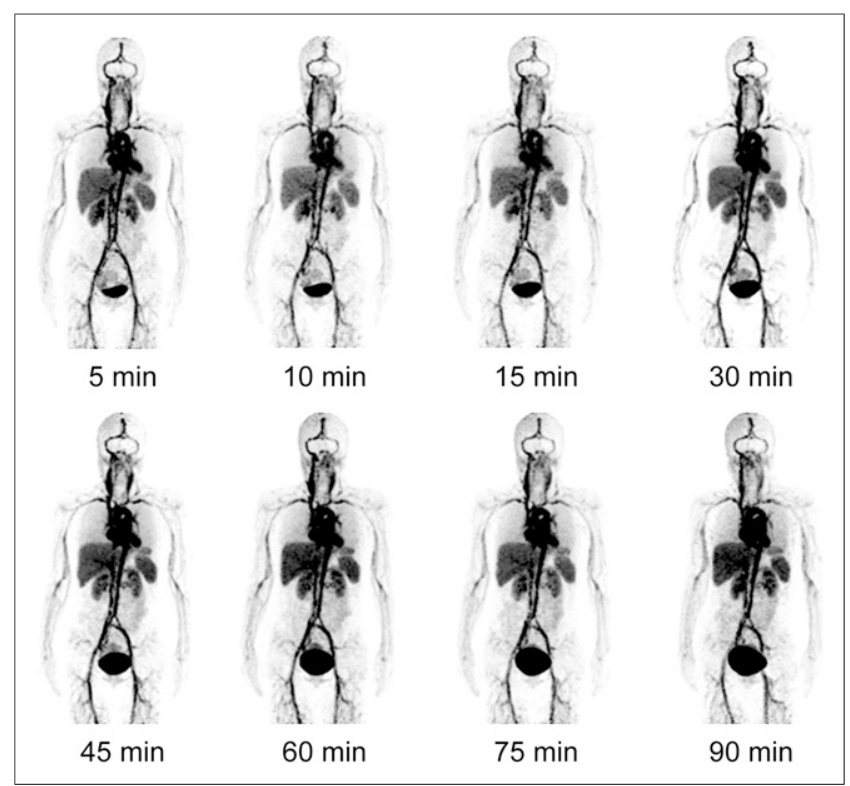

FIGURE 3. Multiple-time-point whole-body maximum-intensity-projection PET images of female healthy volunteer at $5,10,15,30,45,60,75$, and $90 \mathrm{~min}$ after intravenous administration of ${ }^{68} \mathrm{Ga}-\mathrm{NEB}$. 
TABLE 1

Biodistribution of ${ }^{68} \mathrm{Ga}-\mathrm{NEB}$ in Healthy Volunteers (SUV, $n=3$ )

\begin{tabular}{|c|c|c|c|c|c|c|c|c|c|c|}
\hline $\begin{array}{l}\text { Time } \\
\text { (min) }\end{array}$ & Blood & Lung & Liver & Spleen & Kidneys & Stomach & Intestinal & Pancreas & Bone & Muscle \\
\hline 5 & $7.97 \pm 0.15$ & $1.53 \pm 0.15$ & $3.80 \pm 0.44$ & $4.10 \pm 0.36$ & $4.40 \pm 0.26$ & $1.23 \pm 0.15$ & $1.73 \pm 0.25$ & $2.13 \pm 0.40$ & $2.10 \pm 0.26$ & $0.53 \pm 0.21$ \\
\hline 10 & $7.53 \pm 0.25$ & $1.47 \pm 0.29$ & $3.70 \pm 0.61$ & $4.07 \pm 0.47$ & $4.23 \pm 0.49$ & $1.50 \pm 0.20$ & $1.57 \pm 0.12$ & $2.03 \pm 0.15$ & $1.93 \pm 0.12$ & $0.60 \pm 0.10$ \\
\hline 15 & $7.17 \pm 0.35$ & $1.40 \pm 0.17$ & $3.73 \pm 0.59$ & $4.00 \pm 0.52$ & $4.03 \pm 0.50$ & $1.23 \pm 0.12$ & $1.73 \pm 0.12$ & $1.90 \pm 0.26$ & $1.83 \pm 0.15$ & $0.60 \pm 0.17$ \\
\hline 30 & $6.93 \pm 0.21$ & $1.30 \pm 0.17$ & $3.57 \pm 0.46$ & $3.90 \pm 0.61$ & $3.77 \pm 0.29$ & $1.23 \pm 0.06$ & $1.63 \pm 0.15$ & $1.67 \pm 0.15$ & $1.67 \pm 0.15$ & $0.57 \pm 0.12$ \\
\hline 45 & $6.70 \pm 0.26$ & $1.30 \pm 0.20$ & $3.50 \pm 0.52$ & $3.77 \pm 0.57$ & $3.87 \pm 0.32$ & $1.23 \pm 0.06$ & $1.63 \pm 0.06$ & $1.67 \pm 0.15$ & $1.50 \pm 0.20$ & $0.53 \pm 0.06$ \\
\hline 60 & $6.20 \pm 0.17$ & $1.23 \pm 0.06$ & $3.47 \pm 0.47$ & $3.73 \pm 0.42$ & $3.60 \pm 0.10$ & $1.10 \pm 0.00$ & $1.47 \pm 0.15$ & $1.43 \pm 0.15$ & $1.37 \pm 0.06$ & $0.50 \pm 0.00$ \\
\hline 75 & $6.03 \pm 0.12$ & $1.10 \pm 0.17$ & $3.43 \pm 0.49$ & $4.93 \pm 2.10$ & $3.43 \pm 0.15$ & $1.07 \pm 0.12$ & $1.33 \pm 0.21$ & $1.37 \pm 0.21$ & $1.27 \pm 0.12$ & $0.47 \pm 0.06$ \\
\hline 90 & $5.97 \pm 0.15$ & $1.07 \pm 0.12$ & $3.33 \pm 0.58$ & $3.57 \pm 0.55$ & $3.30 \pm 0.20$ & $1.03 \pm 0.15$ & $1.17 \pm 0.32$ & $1.37 \pm 0.31$ & $1.23 \pm 0.15$ & $0.77 \pm 0.64$ \\
\hline
\end{tabular}

veins showed the highest signal density. Vessel branches in and out of major organs and limbs can also be clearly identified. The liver, spleen, and kidneys are also visible, with relatively low activity, whereas the bladder showed high activity.

From 5 to $90 \mathrm{~min}$, no dramatic distribution change was observed, confirming the in vivo stability and long blood-pool retention of ${ }^{68}$ Ga-NEB (Fig. 3). Increased bladder accumulation was observed over time. The average SUVs in the major organs and tissues are listed in Table 1. Although the blood vessels in the brain showed high radioactivity, the normal brain tissue had negligible accumulation of ${ }^{68} \mathrm{Ga}-\mathrm{NEB}$, indicating that the tracer does not cross the blood-brain barrier.

The mean absorbed radiation doses based on multiple-time-point PET images of 3 volunteers were similar to those based on mouse biodistribution data (Table 2). The major organs that received relatively high doses were the kidneys, liver, spleen, and heart wall. The bladder wall also received high exposure due to renal excretion of the radioactivity $(0.0683 \pm 0.0090 \mathrm{mSv} / \mathrm{MBq})$. The whole-body absorbed dose was $0.0151 \pm 0.0001 \mathrm{mSv} / \mathrm{MBq}$, with an effective dose of $0.0179 \pm 0.0003 \mathrm{mSv} / \mathrm{MBq}$.

\section{Differential Diagnosis of Focal Hepatic Lesions}

The widespread use of imaging studies has led to an increase in detection of incidental focal hepatic lesions. Differential diagnosis of malignant and benign solid and cystic liver lesions is important for patient management (27). Among the 11 patients with focal hepatic lesions diagnosed by enhanced CT or MR imaging, 4 were with hemangioma. All hemangiomas showed much higher ${ }^{68} \mathrm{Ga}-\mathrm{NEB}$ signal intensity than the surrounding normal hepatic tissues, whereas no apparent difference between lesions and hepatic tissues was identified on ${ }^{18} \mathrm{~F}$-FDG PET. The lesions were not discernable on regular CT but showed signal enhancement with CT contrast agent (Supplemental Fig. 3). Hepatocellular carcinoma (HCC) showed high tracer uptake on ${ }^{18} \mathrm{~F}$-FDG PET but with big variance from patient to patient. ${ }^{68} \mathrm{Ga}-$ NEB showed consistently lower HCC uptake than normal hepatic tissue (Fig. 4; Supplemental Fig. 4). Similarly, hepatic cysts and neuroendocrine liver metastases also showed a low lesion-to-background ratio with ${ }^{68} \mathrm{Ga}-\mathrm{NEB}$ PET (Fig. 4).

Because of the abundant blood supply, normal liver tissue showed prominent ${ }^{68} \mathrm{Ga}-\mathrm{NEB}$ accumulation with an SUV of $3.73 \pm 0.47$ (Table 3). The SUV of ${ }^{68} \mathrm{Ga}-\mathrm{NEB}$ in hemangiomas $(6.83 \pm 1.38)$ was much higher than that in the surrounding hepatic tissue $(P<0.01)$. All other focal hepatic lesions including HCC, hepatic cysts, and neuroendocrine tumor liver metastases showed negative contrast to hepatic tissues with SUVs of $2.12 \pm 0.16,2.13$, and $2.69 \pm 0.44$, respectively.

\section{DISCUSSION}

This first-in-human study was based on a successful in vivo albumin-labeling strategy with an EB derivative, which formed a complex with serum albumin after intravenous injection (11).

TABLE 2

Absorbed Radiation Doses Per Unit Administered Activity from Intravenous Administration of ${ }^{68} \mathrm{Ga}-\mathrm{NEB}$ ( $\mathrm{mGy} / \mathrm{MBq}, n=3$ )

\begin{tabular}{llc}
\hline \multicolumn{1}{c}{ Target organ } & Mean & SD \\
\hline Adrenals & 0.01393 & 0.00021 \\
\hline Brain & 0.01083 & 0.00072 \\
\hline Breasts & 0.01053 & 0.00055 \\
\hline Gallbladder wall & 0.01530 & 0.00000 \\
\hline Lower large intestine wall & 0.01303 & 0.00057 \\
\hline Small intestine & 0.02523 & 0.00119 \\
\hline Stomach wall & 0.01630 & 0.00026 \\
\hline Upper large intestine wall & 0.01367 & 0.00042 \\
\hline Heart wall & 0.03917 & 0.00045 \\
\hline Kidneys & 0.04617 & 0.00254 \\
\hline Liver & 0.04797 & 0.00697 \\
\hline Lungs & 0.01813 & 0.00153 \\
\hline Muscle & 0.00985 & 0.00074 \\
\hline Ovaries & 0.01337 & 0.00051 \\
\hline Pancreas & 0.02393 & 0.00255 \\
\hline Red marrow & 0.01016 & 0.00041 \\
\hline Osteogenic cells & 0.01590 & 0.00087 \\
\hline Skin & 0.00961 & 0.00050 \\
\hline Spleen & 0.04830 & 0.00548 \\
\hline Testes & 0.01113 & 0.00055 \\
\hline Thymus & 0.01213 & 0.00055 \\
\hline Thyroid & 0.01113 & 0.00055 \\
\hline Urinary bladder wall & 0.06830 & 0.00904 \\
\hline Uterus & 0.01417 & 0.00055 \\
\hline Total body & 0.01507 & 0.00006 \\
\hline Effective dose equivalent & 0.02413 & 0.00071 \\
\hline & 0.01786 & 0.00029 \\
\hline
\end{tabular}




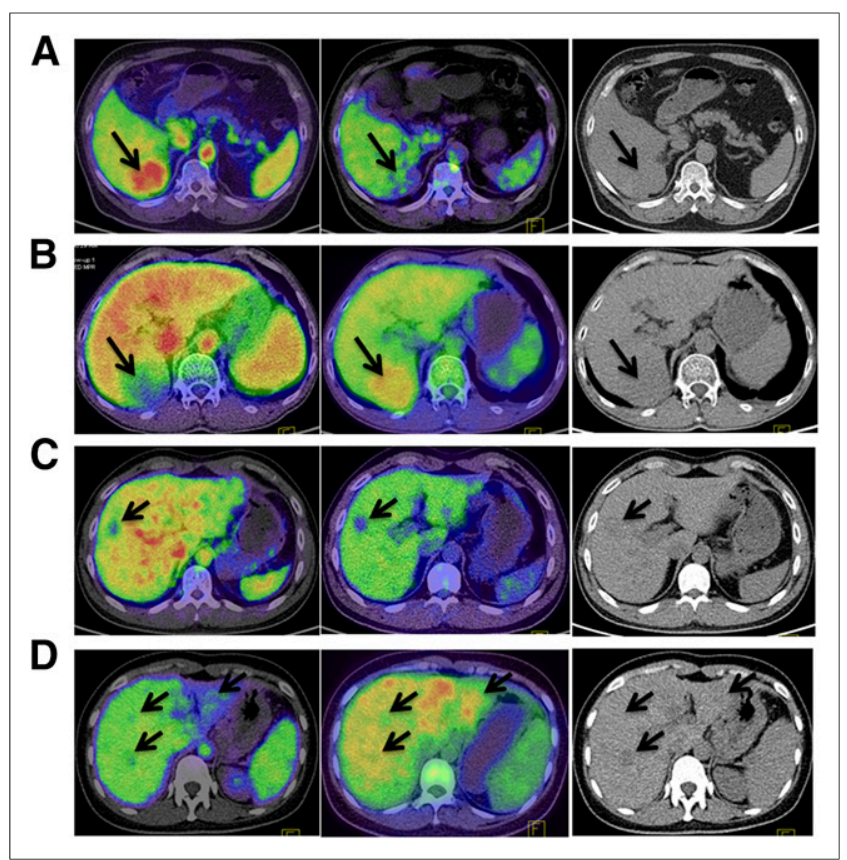

FIGURE 4. Differential diagnosis of focal hepatic lesions using ${ }^{18} \mathrm{~F}-\mathrm{FDG}$ and ${ }^{68} \mathrm{Ga}-\mathrm{NEB}$ PET/CT. (A) Images of hepatic hemangioma: ${ }^{68} \mathrm{Ga}-\mathrm{NEB} \mathrm{PET} / \mathrm{CT}$ (left) shows strong local accumulation of radioactivity with hepatic nodule, whereas ${ }^{18} \mathrm{~F}-\mathrm{FDG}$ PET/CT (middle) shows relatively low local uptake. Nodule is also identified by CT (right) without much signal contrast. (B) Images of hepatic carcinoma. Lesion shows negative contrast compared with surrounding normal hepatic tissue on ${ }^{68} \mathrm{Ga}$ NEB PET/CT (left) whereas increased ${ }^{18} \mathrm{~F}$-FDG uptake is observed with ${ }^{18}$ F-FDG PET/CT (middle). (C) Neuroendocrine tumor liver metastasis. Multiple liver nodules are detected on CT scan (right). ${ }^{68} \mathrm{Ga}-\mathrm{NEB}$ PET/CT (left) shows low uptake in all hepatic nodules, compared with mild to moderate uptake in ${ }^{18} \mathrm{~F}-\mathrm{FDG}$ PET/CT (middle). (D) Hepatic cysts show low uptake of ${ }^{68} \mathrm{Ga}-\mathrm{NEB}$ and ${ }^{18} \mathrm{~F}-\mathrm{FDG}$.

The labeling was efficient without compromising the physiologic behavior of the protein, thus the emitted radioactive signal reflected accurately the in vivo behavior of albumin $(11,28)$. The method also avoided unnecessary cross-contamination from blood products as is the case with labeling of RBCs for blood-pool imaging. For imaging purposes, only a trace amount of the compound was administered so the possible toxicity of the vital dye and potential pulmonary embolism after intravenous injection was completely avoided (29).

Indeed, all healthy volunteers and patients reported no discomfort or adverse clinical events, no elicited acute toxicity, and no allergies. Dosimetry study confirmed the safety with acceptable absorbed doses by critical organs even with multiple injections for 1 patient. With an injected dose of 121-148 MBq (3-4 mCi), a patient would be exposed to a radiation dose of $2.65 \mathrm{mSv}$, which is much lower than the dose limit as set by the Food and Drug Administration (25).

After intravenous injection, most of the radioactivity was retained in blood circulation because of the stable complexation of ${ }^{68} \mathrm{Ga}-\mathrm{NEB}$ with serum albumin. Within a few minutes after tracer injection, the radioactivity reached equilibrium as reflected by the constant high SUV in the blood. A slow but steady clearance of the radioactivity from the blood was observed, which was mainly caused by the turnover of albumin from blood circulation to the interstitial space and slight dissociation of ${ }^{68} \mathrm{Ga}-\mathrm{NEB}$ from albumin. Still, the heart-to-liver ratios at different time points were close to those of in vitro-labeled RBCs and significantly higher than those of in vivo-labeled RBCs (30), confirming the role of ${ }^{68} \mathrm{Ga}-\mathrm{NEB}$ as a blood-pool PET imaging agent.

There are several major applications for a blood-pool imaging agent including evaluation of the cardiac function, detection of vascular anomalies, and localization of neoplasms. Hepatic hemangioma is a vascular anomaly, characterized by multiple vascular channels with a single layer of benign endothelial cells. Consequently, a high level of local accumulation of ${ }^{68} \mathrm{Ga}-\mathrm{NEB}$ was observed, making tumors highly visible against the surrounding normal hepatic tissues. Meanwhile, lesions of HCC, hepatic cyst, and hepatic metastases of neuroendocrine tumors showed negative contrast because of the relatively low accumulation of ${ }^{68} \mathrm{Ga}-\mathrm{NEB}$. Although promising, whether the small number of cases will support the claim that ${ }^{68} \mathrm{Ga}-$ NEB PET would be more specific than CT, MR imaging, and ${ }^{18} \mathrm{~F}-\mathrm{FDG}$ PET in differentiating hemangioma from other focal hepatic lesions remains to be elucidated by future trials with more patients.

The identification of liver lesions is of critical importance because of the increasing incidence of primary hepatic malignancies worldwide and an increase in detection of benign liver lesions by the widespread use of abdomen cross-sectional imaging modalities (31). Although many typical lesions can be detected by traditional imaging tests such as ultrasound, CT, and MR imaging, there remains a challenge to diagnose atypical lesions. For example, hypervascular neuroendocrine tumors often share the same appearance as hemangiomas on MR imaging (32-34). Some atypical hepatic cysts may also show features overlapping with hepatic metastasis from ovarian malignancies (35). The accurate diagnosis of hemangioma with ${ }^{68} \mathrm{Ga}$-NEB PET can avoid unnecessary overtreatment and biopsy, which has the risk of hemorrhage. In combination with ${ }^{18} \mathrm{~F}$ FDG PET, ${ }^{68} \mathrm{Ga}-\mathrm{NEB}$ PET/CT can be of great value for the differential diagnosis of cysts, hemangioma, and other benign hepatic lesions from malignancy, especially in patients with a history of malignancy. It is also predictable that ${ }^{68} \mathrm{Ga}-\mathrm{NEB}$ PET will be helpful in diagnosing hemangioma occurring in other organs.

Although only differential diagnosis of hepatic space-occupying lesions was covered by this pilot clinical study, it is natural to foresee the application of ${ }^{68} \mathrm{Ga}-\mathrm{NEB}$ PET in the evaluation of cardiac function as a blood-pool imaging agent. Although ${ }^{68} \mathrm{Ga}-\mathrm{NEB}$ may not be essential in the detection and staging of malignant tumors, ${ }^{68} \mathrm{Ga}$-NEB PET will give important information of blood supply and vascular density within the lesions. Moreover, different from RBCs, albumin will extravasate in the presence of leaky blood vessel, which is the basis to use EB to evaluate vascular permeability (36). Thus, ${ }^{68} \mathrm{Ga}-\mathrm{NEB}$ PET provides another opportunity to evaluate

TABLE 3

SUVs of ${ }^{18} \mathrm{Ga}-\mathrm{NEB}$ and ${ }^{18} \mathrm{~F}-\mathrm{FDG}$ in Focal Hepatic Lesions $($ Mean \pm SD)

\begin{tabular}{lcc}
\hline \multicolumn{1}{c}{ Hepatic lesions } & ${ }^{18} \mathrm{Ga}-\mathrm{NEB}$ & ${ }^{18} \mathrm{~F}-\mathrm{FDG}$ \\
\hline HCC $(n=7)$ & $2.12 \pm 0.16$ & $5.96 \pm 2.90$ \\
$\begin{array}{c}\text { Neuroendocrine tumor } \\
\text { metastasis }(n=4)\end{array}$ & $2.69 \pm 0.44$ & $2.85 \pm 0.70$ \\
Hemangioma $(n=5)$ & $6.83 \pm 1.38$ & $1.19 \pm 0.19$ \\
Cyst $(n=1)$ & 2.13 & 1.21 \\
$\begin{array}{c}\text { Normal hepatic tissue } \\
(n=11)\end{array}$ & $3.69 \pm 0.53$ & $1.80 \pm 0.41$ \\
\hline
\end{tabular}


tumor angiogenesis and response to antiangiogenic treatment (37). Another reasonable application is to provide a preoperational and intraoperational guide by mapping sentinel lymph nodes by PET and radioactive detector or optical imaging (27).

\section{CONCLUSION}

After successful development of in vivo labeling of albumin with the truncated EB, this is the first report to our knowledge of the clinical applications of ${ }^{68} \mathrm{Ga}-\mathrm{NEB}$ PET. The tracer was demonstrated to be safe in both healthy volunteers and patients without acute toxicity. This small-scale clinical study substantiates the potential of NEB as a blood volume imaging agent in differentiating hepatic hemangioma from other benign or malignant focal hepatic lesions. Easy labeling with different positron emitters, excellent pharmacokinetics, and imaging quality pave a broad way of clinical applications of NEB-derived PET tracers for blood-pool, vascular permeability, and lymphatic imaging.

\section{DISCLOSURE}

The costs of publication of this article were defrayed in part by the payment of page charges. Therefore, and solely to indicate this fact, this article is hereby marked "advertisement" in accordance with 18 USC section 1734. This work was supported by the Intramural Research Program (IRP) of the National Institute of Biomedical Imaging and Bioengineering (NIBIB), National Institutes of Health (NIH). No other potential conflict of interest relevant to this article was reported.

\section{REFERENCES}

1. Rubin GD, Leipsic J, Joseph Schoepf U, Fleischmann D, Napel S. CT angiography after 20 years: a transformation in cardiovascular disease characterization continues to advance. Radiology. 2014;271:633-652.

2. Hasebroock KM, Serkova NJ. Toxicity of MRI and CT contrast agents. Expert Opin Drug Metab Toxicol. 2009;5:403-416.

3. Subramanian KM. Cardiac blood-pool tracers. J Nucl Med. 1991;32:480-482.

4. Kearfott KJ. Absorbed dose estimates for positron emission tomography (PET): $\mathrm{C}^{15} \mathrm{O},{ }^{11} \mathrm{CO}$, and $\mathrm{CO}^{15} \mathrm{O}$. J Nucl Med. 1982;23:1031-1037.

5. Herance JR, Gispert JD, Abad S, et al. Erythrocytes labeled with $\left[{ }^{18} \mathrm{~F}\right] \mathrm{SFB}$ as an alternative to radioactive $\mathrm{CO}$ for quantification of blood volume with PET. Contrast Media Mol Imaging. 2013;8:375-381.

6. Hoffend J, Mier W, Schuhmacher J, et al. Gallium-68-DOTA-albumin as a PET blood-pool marker: experimental evaluation in vivo. Nucl Med Biol. 2005;32: 287-292.

7. Todica A, Brunner S, Boning G, et al. [ $\left.{ }^{68} \mathrm{Ga}\right]$-albumin-PET in the monitoring of left ventricular function in murine models of ischemic and dilated cardiomyopathy: comparison with cardiac MRI. Mol Imaging Biol. 2013;15:441-449.

8. Okazawa H, Yonekura Y, Fujibayashi Y, et al. Measurement of regional cerebral plasma pool and hematocrit with copper-62-labeled HSA-DTS. J Nucl Med. 1996;37:1080-1085.

9. Anderson CJ, Rocque PA, Weinheimer CJ, Welch MJ. Evaluation of copperlabeled bifunctional chelate-albumin conjugates for blood pool imaging. Nucl Med Biol. 1993;20:461-467.

10. Wängler B, Quandt G, Iovkova L, et al. Kit-like ${ }^{18} \mathrm{~F}$-labeling of proteins: synthesis of 4 -(di-tert-butyl $\left[{ }^{18} \mathrm{~F}\right]$ fluorosilyl)benzenethiol $\left(\mathrm{Si}\left[{ }^{18} \mathrm{~F}\right] \mathrm{FA}-\mathrm{SH}\right)$ labeled rat serum albumin for blood pool imaging with PET. Bioconjug Chem. 2009;20: $317-321$.
11. Niu G, Lang L, Kiesewetter DO, et al. In vivo labeling of serum albumin for PET. J Nucl Med. 2014;55:1150-1156.

12. Gibson JG, Evans WA. Clinical studies of the blood volume. I. Clinical application of a method employing the azo dye "Evans Blue" and the spectrophotometer. J Clin Invest. 1937;16:301-316.

13. Spahr PF, Edsall JT. Amino acid composition of human and bovine serum mercaptalbumins. J Biol Chem. 1964;239:850-854.

14. Torabi M, Aquino SL, Harisinghani MG. Current concepts in lymph node imaging. J Nucl Med. 2004;45:1509-1518.

15. Yan J, Manaenko A, Chen S, et al. Role of SCH79797 in maintaining vascular integrity in rat model of subarachnoid hemorrhage. Stroke. 2013;44:1410-1417.

16. Lang L, Li W, Guo N, et al. Comparison study of $\left[{ }^{18} \mathrm{~F}\right] \mathrm{FAl}-\mathrm{NOTA}-\mathrm{PRGD} 2,\left[{ }^{18} \mathrm{~F}\right]$ FPPRGD2, and $\left[{ }^{68} \mathrm{Ga}\right] \mathrm{Ga}-\mathrm{NOTA}-\mathrm{PRGD} 2$ for PET imaging of U87MG tumors in mice. Bioconjug Chem. 2011;22:2415-2422.

17. McBride WJ, D'Souza CA, Sharkey RM, et al. Improved F-18 labeling of peptides with a fluoride-aluminum-chelate complex. Bioconjug Chem. 2010;21:1331-1340.

18. McBride WJ, Sharkey RM, Karacay H, et al. A novel method of F-18 radiolabeling for PET. J Nucl Med. 2009;50:991-998.

19. Baeuerle PA, Gires O. EpCAM (CD326) finding its role in cancer. Br J Cancer. 2007;96:417-423.

20. Carlton JE, Hayes RL. Rapid separation of generator-produced gallium-68 from EDTA eluate. Int J Appl Radiat Isot. 1971;22:44-45.

21. Guide for the Care and Use of Laboratory Animals. Bethesda, MD: National Institutes of Health; 1985. NIH publication 85-23.

22. Sun X, Niu G, Yan Y, et al. Phage display-derived peptides for osteosarcoma imaging. Clin Cancer Res. 2010;16:4268-4277.

23. Lassmann M, Chiesa C, Flux G, Bardies M. EANM Dosimetry Committee guidance document: good practice of clinical dosimetry reporting. Eur J Nucl Med Mol Imaging. 2011;38:192-200.

24. Roivainen A, Kahkonen E, Luoto P, et al. Plasma pharmacokinetics, whole-body distribution, metabolism, and radiation dosimetry of ${ }^{68} \mathrm{Ga}$ bombesin antagonist BAY 86-7548 in healthy men. J Nucl Med. 2013;54:867-872.

25. Mittra ES, Goris ML, Iagaru AH, et al. Pilot pharmacokinetic and dosimetric studies of ${ }^{18}$ F-FPPRGD2: a PET radiopharmaceutical agent for imaging $\alpha v \beta 3$ integrin levels. Radiology. 2011;260:182-191.

26. International Commission on Radiological Protection. The 2007 recommendations of the International Commission on Radiological Protection. ICRP publication 103. Ann ICRP. 2007;37:1-332.

27. Marrero JA, Ahn J, Rajender Reddy K. ACG clinical guideline: the diagnosis and management of focal liver lesions. Am J Gastroenterol. 2014;109:1328-1347, quiz 1348.

28. Wang Y, Lang L, Huang $\mathrm{P}$, et al. In vivo albumin labeling and lymphatic imaging. Proc Natl Acad Sci USA. 2015;112:208-213.

29. Giger M, Baumgartner HR, Zbinden G. Toxicological effects of Evans blue and Congo red on blood platelets. Agents Actions. 1974;4:173-180.

30. Goins B, Phillips WT, Klipper R. Blood-pool imaging using technetium-99mlabeled liposomes. J Nucl Med. 1996;37:1374-1379.

31. Duran R, Ronot M, Kerbaol A, Van Beers B, Vilgrain V. Hepatic hemangiomas: factors associated with $T_{2}$ shine-through effect on diffusion-weighted MR sequences. Eur J Radiol. 2014;83:468-478.

32. Jang HJ, Kim TK, Lim HK, et al. Hepatic hemangioma: atypical appearances on CT, MR imaging, and sonography. AJR. 2003;180:135-141.

33. Caseiro-Alves F, Brito J, Araujo AE, et al. Liver haemangioma: common and uncommon findings and how to improve the differential diagnosis. Eur Radiol. 2007; 17:1544-1554.

34. Silva AC, Evans JM, McCullough AE, Jatoi MA, Vargas HE, Hara AK. MR imaging of hypervascular liver masses: a review of current techniques. Radiographics. 2009;29:385-402.

35. Hussain SM, Semelka RC. Hepatic imaging: comparison of modalities. Radiol Clin North Am. 2005;43:929-947.

36. Ronaldson PT, Davis TP. Targeting blood-brain barrier changes during inflammatory pain: an opportunity for optimizing CNS drug delivery. Ther Deliv. 2011;2:1015-1041.

37. McDonald DM, Choyke PL. Imaging of angiogenesis: from microscope to clinic. Nat Med. 2003;9:713-725. 